\title{
Experimental Evidence in Support of the Possibility That the Local Space of Earth Is Confined
}

\author{
Shlomo Barak ${ }^{1}$, Arie Zigler ${ }^{2} \&$ Jenya $_{\text {Papeer }}^{2}$ \\ ${ }^{1}$ Taga Innovations 16 Beit Hillel St. Tel Aviv 670017, Israel \\ ${ }^{2}$ Racah Institute of Physics, Hebrew University, Jerusalem, Israel \\ Correspondence: Shlomo Barak, Taga Innovations 16 Beit Hillel St. Tel Aviv 670017, Israel. E-mail: \\ shlomo@tagapro.com
}

Received: May 5, 2019

Accepted: May 23, 2019

Online Published: July 15, 2019

doi:10.5539/apr.v11n4p7

URL: http://dx.doi.org/10.5539/apr.v11n4p7

\begin{abstract}
We have measured variations in the times of flight of 25 fsec laser pulses, along a given distance from $A$ to $B$, in different directions in space. Flights from A to B are termed one-way versus two-way - from A to B and back to A. The isotropy obtained, despite the motion of Earth in global space, supports the possibility that the local space of Earth, and probably that of other stars, is confined - captured inside the sphere of the star. To maintain space continuity, despite this confinement, space must necessarily be an elastic fluid, as Einstein, for a different reason, concluded in 1939.

In addition, we wonder if this confinement of a local space in a star or galaxy explains its non-participation in the Hubble expansion of space.

Our experiment used an auto-correlation technique that dispels the need for clocks, which in one-way measurements require a problematic synchronization scheme.
\end{abstract}

Keywords: Space, Light velocity, General Relativity (GR), Compressible Fluid

\section{Introduction}

In the past, the understanding was that light doesn't need a medium to propagate through (Einstein, 1905); no need for an Aether. Today, however, it is clear that electromagnetic waves are the vibrations of an electromagnetic medium, which might be space itself as some are arguing.

Mutual scattering of light beams in free space, according to the electromagnetic theory, is impossible, since the equations of the theory are linear. This, however, accrues in free space with high-intensity laser beams (ATLAS Collaboration, 2019). This phenomenon can only be attributed to the nonlinearity of an existing medium, in which the beams interact. Thus, logically you have to attribute the nonlinearity whether to an electromagnetic medium or space itself. Note also, that this medium, in classical physics and according to GR, follows space topology, as evident from the bending of light. Hence, the rest or motion of our experimental system relative to the electromagnetic medium is its rest or motion relative to space.

Therefore, the isotropy obtained in our experiment compels us to consider the possibility that, despite the Earth's celestial motions in the global space, the local space of Earth is confined, and space in general is an elastic fluid a compressible liquid as Einstein concluded (Einstein, 1939, p. 922).

Light velocity is the distance the light passes divided by the time it takes to pass this distance. Intrinsic light velocity, however, is the velocity of the electromagnetic medium's light wave, which is dependent on its permittivity and permeability.

\section{Isotropy and Anisotropy in the One-Way Times of Flight}

For Earth sliding through space (as if space is stationary and each Earth's elementary particle moves through it) one-way times of flight of laser pulses, measured by stationary instrumentation on Earth, should be anisotropic. Isotropy, however, could only be a result of the instrumentation being stationary relative to space. This can happen if the local space of Earth is confined.

A one-way speed of light measurement requires clocks and a synchronization scheme (Roberts \& Schleif, 2006). 
It is possible, however, to measure a variation in the one-way speed of light without clocks, using an autocorrelation technique (Gubner, 2006), as we did.

We show below that for Earth sliding through space a variation in the time of flight of the counter-propagating light pulses, in the sliding direction (or opposite to it), is supposed to yield the sliding velocity. This velocity could have been expected to be the Earth's velocity in the Cosmological Microwave Background (CMB) frame (Muller, 1977).

However, aligning our system in various directions in the celestial sphere, yielded isotropy in our measurements. Isotropy in a one-way light velocity measurement, using different techniques, was also reported recently by Ahmed et al. (2013), and Gurzadyan and Margaryan (2018).

Let the velocity relative to space of our stationary laboratory on Earth, $\mathbf{v}$, be the velocity of Earth in the CMB frame. For the following derivation let this velocity be a hypothetical sliding through space velocity.

For $\mathrm{v}<<\mathrm{c}$ the time of flight, $\Delta \mathrm{t}$, of a laser pulse along a distance $\mathrm{L}$ in the direction of $\mathbf{v}$, is:

$$
\Delta \mathrm{t}_{1}=\mathrm{L} /(\mathrm{c}-\mathrm{v}) \sim(\mathrm{L} / \mathrm{c})(1+\mathrm{v} / \mathrm{c})
$$

We ignore any possible Lorentz contraction of $\mathrm{L}$, for any reason, since it is $(\mathrm{v} / \mathrm{c})^{2}$ dependent, whereas the measurement of $\Delta t$ depends on (v/c), see equation (1). For the direction opposite to $\mathbf{v}$ the time of flight is (2) and the times difference, is (3):

$$
\begin{gathered}
\Delta \mathrm{t}_{2}=\mathrm{L} /(\mathrm{c}+\mathrm{v}) \sim(\mathrm{L} / \mathrm{c})(1-\mathrm{v} / \mathrm{c}) \\
\Delta \mathrm{t}=\Delta \mathrm{t}_{1}-\Delta \mathrm{t}_{2}=2 \mathrm{~L} / \mathrm{c}^{2} \cdot \mathrm{v}
\end{gathered}
$$

A measurement of the variation in the one-way time of flight of two counter propagating pulses in the direction of $\mathrm{v}$, along a given distance, is thus $\mathrm{v} / \mathrm{c}$ dependent.

Our isotropic result: $\Delta t=0$ means, therefore, that the velocity of our laboratory relative to space is: $v=0$. This could only be a result of the local space of Earth being confined.

This result complies with the theory of Special Relativity, since it implies that the velocity of light is constant. Motion of our experimental system, however, might yield anisotropic results.

\section{The Experiment}

Figure 1 shows the measurement set-up. The goniometer is a circular plate $100 \mathrm{~cm}$ in diameter mounted on a tripod. The plate is balanced horizontally and the optical bench, mounted on top of it, can rotate around its center and point at different directions in space. The $0^{0}$ and the $180^{\circ}$ marks on the circumference of the goniometer point from south to north, with $0.2^{0}$ accuracy.

Laser pulses from a $25 \mathrm{fsec}$ Ti: Sapphire laser are delivered to the optical bench, see Figure 2. The laser is a $20 \mathrm{fsec}$ Spectra Physics Tsunami oscillator, followed by a regenerative amplifier. The pulse energy, at a wavelength of $798 \mathrm{~nm}$, is $3 \mathrm{~mJ}$, and the repetition rate is $10 \mathrm{~Hz}$. The laser beam diameter is $\sim 0.8 \mathrm{~cm}$. Our system superimposes two laser beams on a Barium Borate (beta- $\mathrm{BaB}_{2} \mathrm{O}_{4}, \mathrm{BBO}$ ) crystal that serves as our autocorrelator. The $600 \mathrm{~mm}$ optical rod, constructed of two $300 \mathrm{~mm}$ rods, serves as an optical delay line that ensures the simultaneous arrival of the two pulses to point $\mathrm{C}$, Figure 2, on the first arm and at point $\mathrm{G}$ on the second arm.

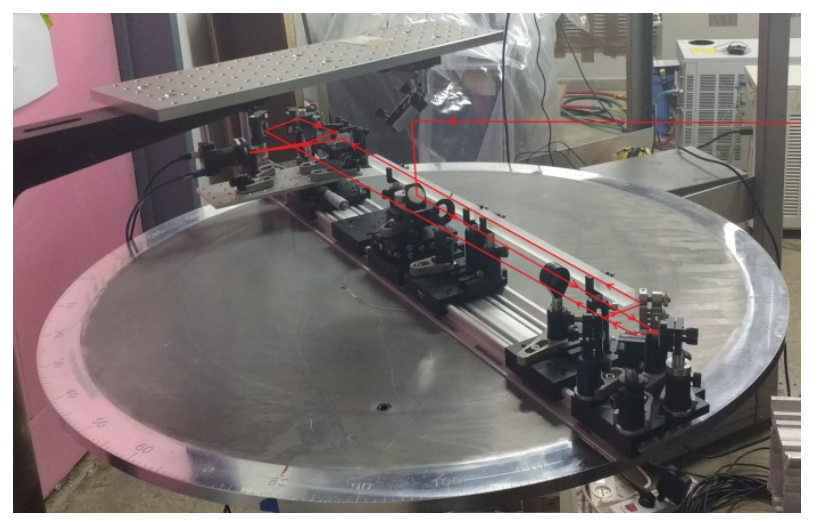

Figure 1. The Goniometer \& Optical Bench. The red line represents the trajectory of the laser pulse with arrows indicating the direction of propagation of the pulse 
The rods are: Schott BK7 glass, cross-section $12 \mathrm{~mm}$, index of refraction $\mathrm{n}=1.51$ for $\lambda=798 \mathrm{~nm}$. We ignore the variation in the refractive index of the glass $\mathrm{n}$, due to a possible Lorentz contraction, since this is $(\mathrm{v} / \mathrm{c})^{2} \mathrm{dependent}$. The time interval $\Delta \mathrm{t}$ (rod) of a pulse moving along the rod, being a moving medium in space, is: $\Delta \mathrm{t}(\mathrm{rod})=\mathrm{L} /(\mathrm{c}$ $/ \mathrm{n}+\mathrm{v}$ ), using $+\mathrm{v}$ for pulses moving in the direction of the system from A to B, or $-\mathrm{v}$ for pulses moving in the opposite direction.

The laser beam pulses are delivered to the optical bench vertically and enter the bench at point $\mathrm{O}$ on a mirror mounted on the rotation axis of the bench. From this mirror the pulses are delivered to point P. At point A, a 50:50 beam splitter delivers one half of the beam to point $\mathrm{C}$ and the other half via point $\mathrm{I}$ and through a glass rod to $\mathrm{G}$. The distance $\mathrm{AB}(\mathrm{AB}=\mathrm{AC})$ was chosen so that the optical path $\mathrm{ABC}=\mathrm{AIG}$. The two beams pass through equal optical lengths and in the same direction, hence the times of flight along these paths are equal - points $\mathrm{C}$ and $\mathrm{G}$ are reached simultaneously regardless of the bench's direction in space.

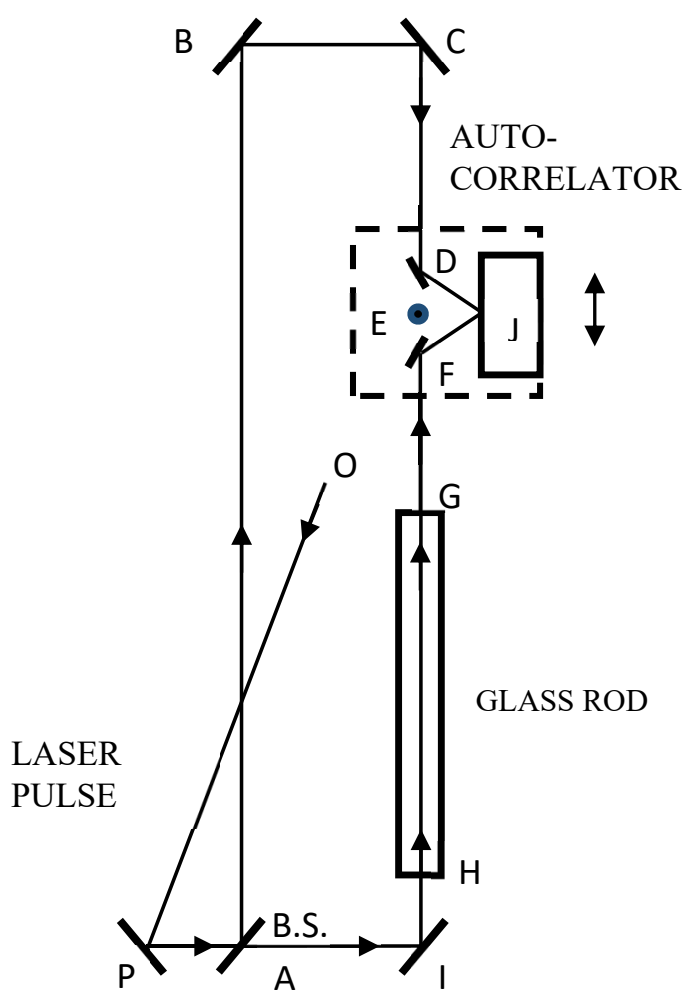

Figure 2. The Optical Bench

The micrometer-controlled stage (dashed square) that carries the autocorrelator is located in approximately the middle of CG. Autocorrelation is achieved when the time of flight along paths ACE and AGE, taking in account the effect of velocity, are equal.

The scanning of the celestial sphere was done by orienting the optical bench on the goniometer at different angels for 12 hours at each angle and letting the Earth rotation do the scanning.

The outcome was an isotropy in the times of flight of the pulses.

The autocorrelation of the $25 \mathrm{fsec}$ laser pulses is obtained within $\Delta \mathrm{l}=+/-20 \mu \mathrm{m}$ of the stage movement, which is a time difference of $\Delta \mathrm{t}^{\prime}=\Delta \mathrm{l} / \mathrm{c}=6.6 \cdot 10^{-14} \mathrm{sec}$. For Earth sliding through space the arrival time difference (3) for $2 \mathrm{~L}=\mathrm{CE}+\mathrm{GE}=30 \mathrm{~cm}$ gives the minimal velocity that could have been detected, which is: $\mathrm{v}=\Delta \mathrm{t}^{\prime} \cdot \mathrm{c}^{2} / 2 \mathrm{~L}=22$ $\mathrm{km} / \mathrm{sec}$.

The velocity of Earth in the CMB frame, however, is $\sim 340 \mathrm{~km} / \mathrm{sec}$ and around the Sun $30 \mathrm{~km} / \mathrm{sec}$.

\section{Summary}

We have obtained isotropy in one-way times of flight of laser pulses. We explain this isotropy by the local space of Earth being confined. Therefore, we have to conclude that to maintain space continuity it is also possible that 
space is an elastic fluid.

\section{Acknowledgements}

We would like to thank Mr. Emanuel Binnun and Dr. Igal Pat-El for the astronomical calculations, Mr. Avshalom Harat for the mechanical work and Mr. Roger M. Kaye for his linguistic contributions and technical assistance.

\section{Conflict of interests}

The authors declare that there is no conflict of interests regarding the publication of this paper.

\section{References}

Ahmed et al. (2013, October 4). Results of a one-way experiment to test the Isotropy of the speed of light.

ATLAS Collaboration. (2019, March 17). ATLAS observes light scattering off light.

Einstein, A. (1905). On the Electrodynamics of Moving bodies. Dover, 1923.

Einstein, A. (1939). On a Stationary System with Spherical Symmetry Consisting of Many Gravitating Masses. The Annals of Mathematics, Second Series, 40(4).

Gubner, J. A. (2006). Probability and Random Processes for Electrical and Computer Engineers. Cambridge University Press. ISBN 978-0-521-86470-1.

Gurzadyan, V. G., \& Margaryan, A. T. (2018, July 23). The light speed vs the observer: The Kennedy-Thorndike test from GRAAL-ESRF.

Muller, R. A. (1977, May). The Cosmic Background Radiation and the New Aether Drift. Sci. Am., 64.

Roberts, T., \& Schleif, S. (2006). Relativity FAQ. One-Way Tests of Light-Speed Isotropy.

\section{Copyrights}

Copyright for this article is retained by the author(s), with first publication rights granted to the journal.

This is an open-access article distributed under the terms and conditions of the Creative Commons Attribution license (http://creativecommons.org/licenses/by/4.0/). 